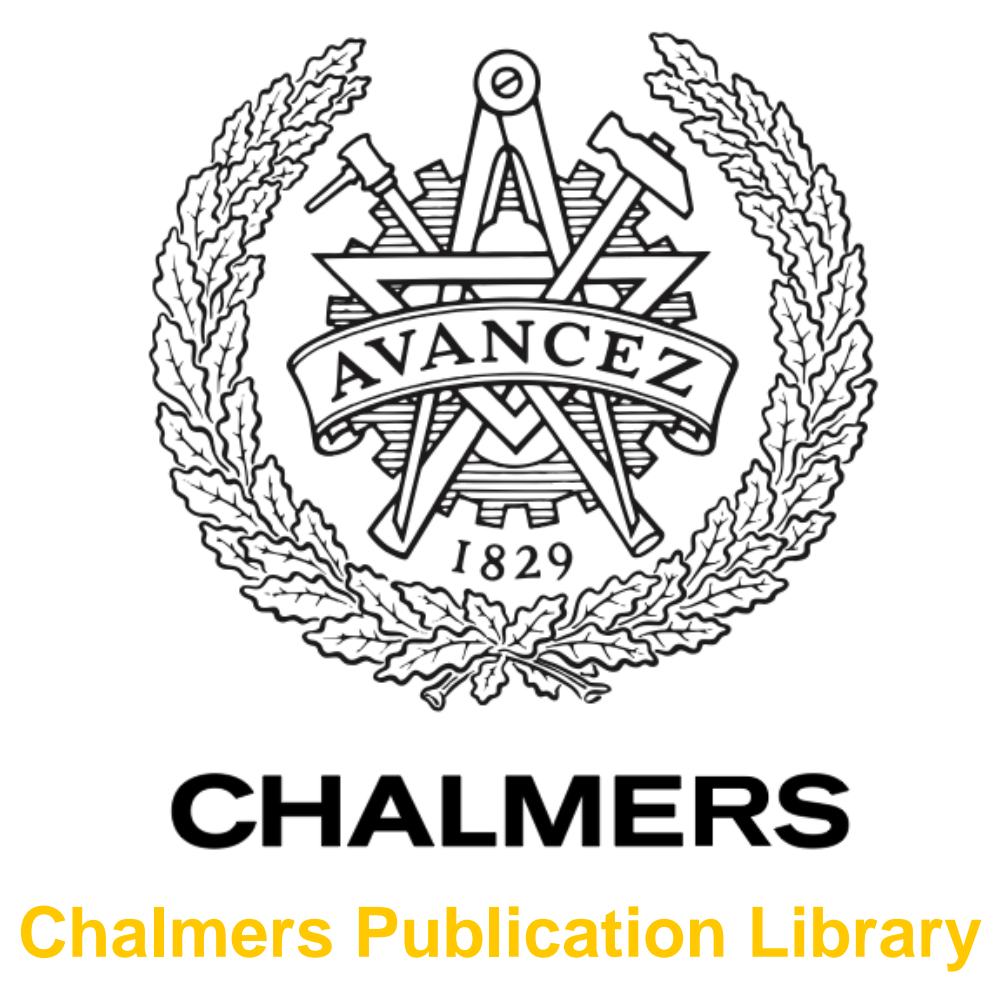

\title{
Coverage Analysis for Millimeter Wave Uplink Cellular Networks with Partial Zero- Forcing Receivers
}

This document has been downloaded from Chalmers Publication Library $(\mathrm{CPL})$. It is the author's version of a work that was accepted for publication in:

15th International Symposium on Modeling and Optimization in Mobile, Ad Hoc, and Wireless Networks (WiOpt)

Citation for the published paper:

Fang, C. ; Makki, B. ; Svensson, T. (2017) "Coverage Analysis for Millimeter Wave Uplink Cellular Networks with Partial Zero-Forcing Receivers". 15th International Symposium on Modeling and Optimization in Mobile, Ad Hoc, and Wireless Networks (WiOpt) pp. Article no. 7959947.

http://dx.doi.org/10.23919/WIOPT.2017.7959947

Downloaded from: http://publications.lib.chalmers.se/publication/249519

Notice: Changes introduced as a result of publishing processes such as copy-editing and formatting may not be reflected in this document. For a definitive version of this work, please refer to the published source. Please note that access to the published version might require a subscription. 


\title{
Coverage Analysis for Millimeter Wave Uplink Cellular Networks with Partial Zero-Forcing Receivers
}

\author{
Chao Fang, Behrooz Makki, and Tommy Svensson, \\ Department of Signals and Systems, Chalmers University of Technology, Gothenburg, Sweden \\ emails: \{fchao, behrooz.makki, tommy.svensson\}@ chalmers.se
}

\begin{abstract}
Cellular networks operating at millimeter wave (mmWave) frequencies are able to achieve multi-gigabit-persecond data rates due to the large bandwidth available. However, the data transmission range will be shorter and significant signal power difference will be observed between line-of-sight (LOS) and non-line-of-sight (NLOS) links. This paper considers interference management and useful signal enhancement in the uplink transmission of small-cell mmWave networks. Taking blockages into account, we analyze the coverage performance of the partial-zero-forcing (PZF) receiver which utilizes a number of antennas to cancel out the strongest uplink interferers and uses the rest of the antennas for boosting the useful signal. Using stochastic geometry, we derive analytical expressions for the coverage probability of the PZF receiver under a LOS probability function based path loss model. For a broad range of parameter settings, the maximum coverage probability is achieved by using most antennas for array gain and only canceling a few strongest interferers. Particularly, compared to zero-forcing, the PZF scheme can improve the coverage probability significantly.

Index terms-Millimeter-wave, uplink, partial-zero-forcing receiver, stochastic geometry.
\end{abstract}

\section{INTRODUCTION}

The huge amount of data which will be handled by wireless networks in the near future put high requirements on data rates, coverage etc. Due to the large spectrum available from 6-100 GHz, millimeter-wave (mmWave) communication opens up new opportunities to achieve multi-gigabit-per-second data rates as well as to serve large number of user devices. Although mmWave communications faces challenges such as high attenuation and hardware constraints, recent research [1][3] showed that mmWave communications is a promising technology for the fifth generation (5G) cellular networks. This is because the small cell deployment and the directional beamforming can be used to facilitate the limited transmission range of mmWave signals and achieve appropriate interference management.

Compared to the downlink transmission, applications such as social networking and high-definition multimedia streaming will make the uplink transmissions more demanding, thus the ratios between the uplink and the downlink will vary much between applications [4]. In mmWave uplink transmissions, because of limited transmit power, limited beamforming capabilities at mobile users and high attenuation of mmWave signals, the uplink transmission range should be relatively short. Therefore, it is preferred that small base stations (BSs) are densely deployed in traffic-intense areas, so that a mobile user can be served in the uplink by a small BS in the immediate vicinity. As a result of the unstructured small cell networks and the large number of users, mobile users served by different BSs would have to share the same uplink frequency resource, thus causing inter-cell uplink interference to each other. Furthermore, the sensitivity to blockage of mmWave uplink signals can lead to different path loss between line-ofsight (LOS) and non-line-of-sight (NLOS) channels [1], [5]. In order to circumvent the high uplink interference due to users in a highly populated area and enhance the desired uplink signal due to blockage, multiple antennas should be employed at the BSs to provide array gain or suppress the interference.

The performance of unstructured cellular networks has been extensively analyzed using stochastic geometry [6], [7]. For mmWave cellular networks, T. Bai et al. proposed a random building distribution to analyze the blockage effects and found that the LOS probability decreases exponentially with the link distance [8]. Moreover, M.N. Kulkarni et al. used real building locations to model the blockage effects and studied the signal-to-interference-plus-noise ratio (SINR) characterization [9]. Modeling the BSs as a homogeneous Poisson point process (PPP), the authors in [10] gave the downlink coverage probability considering antenna beamforming gains under a probabilistic LOS/NLOS model. In [11], the probabilistic LOS/NLOS model was compared with the 3GPP-proposed LOS probability function, then the coverage probability, the spectral efficiency and the area spectral efficiency were derived in dense small-cell networks.

\section{A. Contributions}

In this paper, we analyze the coverage probability of uplink mmWave small-cell networks where users are equipped with single antennas and BSs utilize multiple antennas to enhance uplink signals and reduce interference. The results are presented in the cases with the PZF receiver incorporated with a LOS probability function based path loss model. With PZF, the coverage probability is improved by enhancing the useful signal with a subset of the antennas' degree-of-freedoms and using the rest of the degrees-of-freedom for interference cancellation. We derive exact closed-form expression (Theorem 1) as well as an upper bound (Theorem 2) on the coverage probability of the PZF-based receiver. Moreover, we analyze the effect of different fading distributions (Rayleigh fading 
and Rician fading), the blockage probability and the transmit power on the coverage probability. Our work provides an analytical tool to predict how to best utilize antennas in dense mmWave networks.

The results show that there exists an optimal number of canceled interferers maximizing the coverage probability. Particularly, for a broad range of parameter settings, it is optimal to use most of the antennas to exploit the array gain, because it is likely that there exists only a few large interference signals due to the blockage effects. The optimal number of antennas for interference cancellation depends on channel parameters which could cause increased interference powers, such as the fading distribution or the transmitter power.

\section{System MODEL}

We consider a network where mobile users are distributed according to a Poisson point process (PPP) $\Phi$ with density $\lambda$ [6]. In harmony with [12], we assume that at least one randomly chosen BS from a set of BSs falls into the Voronoi cell of any uplink user and the unchosen BSs do not have an uplink user scheduled. Note that, in general, the homogeneous PPP distribution can not be directly applied for modeling users' locations in the uplink transmission, as there could be no BSs associated to an uplink user, a Poisson-Voronoi perturbed lattice is usually used to model users' locations [13]. However, for tractability, we adopt this assumption, as it makes the users' positions and their associated BSs independent and allows us to characterize the uplink interference at a random point in $\mathbb{R}^{2}$ [6]. It has been shown that, the joint probability densities of the distances of two users to their associated BSs in two neighboring Voronoi cells are similar to that without this assumption [12].

We consider the case that the mobile users served by the same BS use orthogonal frequency division multiple access for uplink transmissions so that there is no intra-cell uplink interference at each BS. However, because the same frequency band could be reused in adjacent BSs, there is uplink intercell interference. That is, if a user $x_{0}$ is transmitting to the serving $\mathrm{BS} B_{0}$, there are other active users $x_{k} \in \Phi \backslash x_{0}, k>0$ from adjacent cells causing interference to $B_{0}$. Without loss of generality, $B_{0}$ is assumed to be placed at the origin of a $2 \mathrm{D}$ plane. The channel model and the receiver technique are given as follows.

Path Loss Model: We consider a path loss model which defines a LOS probability function and calculates the path loss of a LOS/NLOS link as [10], [11], [15]

$$
\operatorname{PL}(d)=\mathbb{I}\left(p_{L}(d)\right) C_{L} d^{-\alpha_{L}}+\left(1-\mathbb{I}\left(p_{L}(d)\right)\right) C_{N} d^{-\alpha_{N}} .
$$

Here, $\mathbb{I}\left(p_{L}(d)\right)$ follows the Bernoulli distribution with success probability $p_{L}(d), d$ is the distance from a user to $B_{0}$, $\alpha_{L}, \alpha_{N}$ and $C_{L}, C_{N}$ are the path loss exponents and the carrier frequency dependant constants for LOS and NLOS links, respectively. The LOS probability function is given by $p_{L}(d)=e^{-\beta d}$, where $\beta$ is chosen according to the distribution of blockages. Typical values of $\beta$ for different scenarios can be estimated using measurements [8], [15]. Note that, for tractability, this path loss model assumes independent blockages, the dependency in blockage of adjacent users is left for future work.

Uplink Cell Association : We assume all users transmit with fixed power $P$. Although power control is not specifically studied in this work, the effect of the transmit power on the coverage probability is shown in Section IV. Also, it is possible to include the fractional power control proposed in [12], [14] into our model, hence power control is considered for future work. We further assume each user chooses its serving BS according to the received signal power averaged over fading, and only scheduled uplink users are considered, so that there are no users that are not served by a BS.

Transmit and Receive Structure: The BS is assumed to have $N$ omni-directional antennas and use $K, 0 \leq K \leq$ $N-1$, degrees of freedom of the antennas for canceling the interference. The canceled signals are the $K$ strongest interferers with smallest path loss. The remaining $N-K$ degrees of freedom are used for enhancing the useful signal power. We limit our analysis to single-antenna users and multiple-antenna BSs. This is motivated by the fact that the PZF receiver has the advantage of low power consumption at the user side, low complexity and analytical tractability compared to other receivers such MMSE receivers [16], [17]. Investigations on using multiple antennas at both sides and complexity/performance comparisons with hybrid/analogue beamforming will be considered in the future.

Let the channel vector for user $x_{i}$ be $\boldsymbol{h}_{i} \sim \mathcal{C N}(0, \boldsymbol{I})$ and neglect the channel correlation. The PZF receiver computes a receive filter $\boldsymbol{v}_{0}$ that is in the direction of the projection of $\boldsymbol{h}_{0}$ on the null space of $\left(\boldsymbol{h}_{1}, \ldots, \boldsymbol{h}_{K}\right)$ [16], [17], where $\boldsymbol{h}_{0}$ is the fading channel vector for the serving user and $\boldsymbol{h}_{1}, \ldots, \boldsymbol{h}_{K}$ are the fading channel vectors corresponding to the $K$ largest interferers. Thus, the receiver filter is given by

$$
\boldsymbol{v}_{0}=\frac{\boldsymbol{Q} \boldsymbol{Q}^{H} \boldsymbol{h}_{0}}{\left\|\boldsymbol{Q}^{H} \boldsymbol{h}_{0}\right\|}
$$

where $Q$ is an orthonormal basis of the null-space of $\left(\boldsymbol{h}_{1}, \ldots, \boldsymbol{h}_{K}\right)$ and $\boldsymbol{Q}^{H}$ denotes the conjugate transpose of $\boldsymbol{Q}$. Note that, if $K=0$, then $\boldsymbol{v}_{0}=\frac{\boldsymbol{h}_{0}}{\left\|\boldsymbol{h}_{0}\right\|}$, and the PZF receiver is reduced to the conventional MRC receiver in which the desired signal power is maximized. If $K=N-1, \mathrm{PZF}$ operates at full interference cancellation, which we refer to as the zero forcing $(\mathrm{ZF})$ receiver.

Received SINR: The received useful signal and the interference signals at the BS are shown in Fig. 1. Denoting the distance from $B_{0}$ to all users by $\left\|x_{k}\right\|, k \geq 0$, the index $k$ is ordered such that $\left\|x_{0}\right\|^{\alpha_{0}} / C_{0} \leq\left\|x_{1}\right\|^{\alpha_{1}} / C_{1} \leq \cdots$, where the values of $\alpha_{k}$ and $C_{k}$ depend on if the link is LOS or NLOS. Based on the above system model, the received SINR at the $\mathrm{BS}$ is given by

$$
\mathrm{SINR}=\frac{y_{0}^{-1}\left|\boldsymbol{v}_{0}^{H} \boldsymbol{h}_{0}\right|^{2}}{\sum_{k=K+1}^{\infty} y_{k}^{-1}\left|\boldsymbol{v}_{0}^{H} \boldsymbol{h}_{k}\right|^{2}+1 / \mathrm{SNR}_{L}},
$$

where $\mathrm{SNR}_{L}=C_{L} P / \sigma^{2}, \sigma^{2}$ is the additive noise power, $P$ is the transmit power and $y_{i}=C_{L}\left\|x_{i}\right\|^{\alpha_{i}} / C_{i}$. The $K$ 


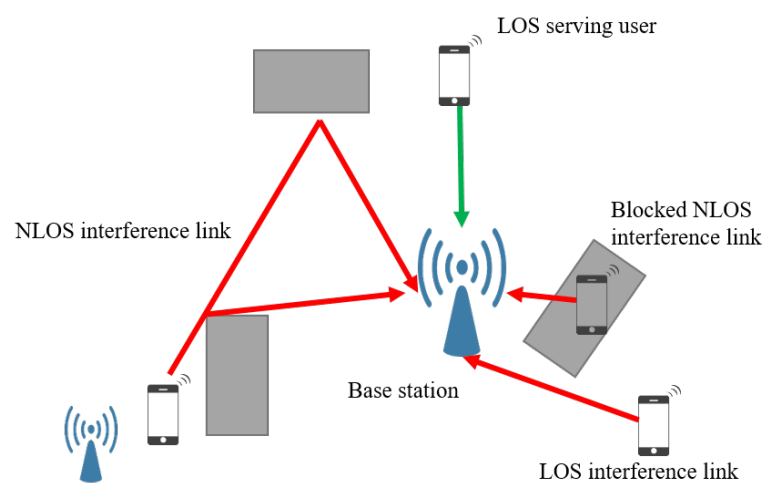

Figure 1. System model for dense uplink mmWave networks. Due to the blockage effects, the nearest user may not have the strongest receive power at the base station.

strongest interference signals are canceled, since $\boldsymbol{v}_{0}$ is orthogonal to $\left(\boldsymbol{h}_{1}, \ldots, \boldsymbol{h}_{K}\right)$. Note that the SINR is random due to the interfering user locations, the LOS/NLOS condition of each link and the output channel distribution. According to our cell association strategy, the serving user is chosen by $x_{0}: \max _{i \geq 0} y_{i}^{-1}$. Due to the presence of LOS and NLOS links, $B_{0}$ may not necessarily serve the nearest user, because a LOS user can result in a smaller path loss than a nearby NLOS user. Similarly, the $K$ strongest interferers may not necessarily be the $K$ nearest interferers.

\section{UPLINK COVERAGE IN MMWAVE NETWORKS}

In this section, we study the coverage probability for uplink mmWave cellular networks using the PZF receiver. We first derive the Laplace transform of the interference (Lemma 1) and then find the closed-form coverage probability expressions based on the Laplace transform (Theorem 1-2).

\section{A. Laplace Transform of the Interference Power}

Let the LOS/NLOS nodes denote users with LOS/NLOS transmissions to $B_{0}$. Due to the independence of the blockage, each node in $\Phi$ is independently determined to be a LOS node or an NLOS node according to the LOS probability $p_{L}(d)$. From the Thinning Theorem [6, Theorem 2.36], $\Phi$ can be divided into two independent inhomogeneous PPPs $\Phi_{L}$ and $\Phi_{N}$ with density $\lambda_{L}(x)=\lambda p_{L}(\|x\|)$ and $\lambda_{N}(x)=\lambda\left(1-p_{L}(\|x\|)\right)$, respectively [10], [11]. Because $y_{k}=C_{L}\left\|x_{k}\right\|^{\alpha_{k}} / C_{k}, x_{k} \in \Phi$ is a function mapping $x_{k}$ in $\mathbb{R}^{2}$ to $y_{k}$ in $\mathbb{R}$, according to the Mapping theorem [6, Theorem 2.34], $y_{k}$ forms an inhomogeneous PPP with intensity measure $\Lambda[(0, y)]=\Lambda_{L}[(0, y)]+\Lambda_{N}[(0, y)]$, where

$$
\begin{aligned}
& \Lambda_{L}[(0, y)]=\frac{2 \pi \lambda}{\beta^{2}}\left[1-\left(1+\beta y^{\eta_{L}}\right) e^{-\beta y^{\eta_{L}}}\right] \\
& \Lambda_{N}[(0, y)]=\pi \lambda \gamma^{2} y^{2 \eta_{N}}-\frac{2 \pi \lambda}{\beta^{2}}\left[1-\left(1+\beta \gamma y^{\eta_{N}}\right) e^{-\beta \gamma y^{\eta_{N}}}\right],
\end{aligned}
$$

and density function $\lambda(y)=\frac{\partial \Lambda(y)}{\partial y}=\lambda_{L}(y)+\lambda_{N}(y)$, where

$$
\begin{aligned}
\lambda_{L}(y) & =2 \pi \lambda \eta_{L} y^{2 \eta_{L}-1} e^{-\beta y^{\eta_{L}}} \\
\lambda_{N}(y) & =2 \pi \lambda \eta_{N} \gamma^{2} y^{2 \eta_{N}-1}\left(1-e^{-\beta \gamma y^{\eta_{N}}}\right),
\end{aligned}
$$

with $\eta_{L}=1 / \alpha_{L}, \eta_{N}=1 / \alpha_{N}$ and $\gamma=\left(C_{N} / C_{L}\right)^{\eta_{N}}$. The derivation of these density measures and the density functions is similar to the proof of [18, Lemma 7]. The only difference is that we include the frequency dependent constants $C_{L}$ and $C_{N}$.

Denoting the inhomogeneous PPP of $y_{k}$ by $\Phi_{y}$, it follows that $y_{0}$ is the nearest point to 0 in $\Phi_{y}$ and $y_{k}$ is the $k$-th nearest point to $y_{0}$. We can obtain the PDF of $y_{i}$ following similar procedures as in deriving the distribution of the distance to the $i$-th neighbor [19], and obtain the PDF of $y_{0}$

$$
f_{y_{0}}(y)=\lambda(y) e^{-\Lambda[(0, y)]},
$$

and the PDF of $y_{k}$ conditioned on $y_{0}$

$f_{y_{k} \mid y_{0}}(y)=\lambda(y) e^{\Lambda\left[\left(0, y_{0}\right)\right]-\Lambda[(0, y)]} \frac{\left(\Lambda[(0, y)]-\Lambda\left[\left(0, y_{0}\right)\right]\right)^{k-1}}{\Gamma(k)}$,

where $\Gamma(k)=(k-1)$ ! for all positive integers $k$.

Denoting the total interference power by $I=\sum_{k=K+1}^{\infty} y_{k}^{-1}\left|\boldsymbol{v}_{0}^{H} \boldsymbol{h}_{k}\right|^{2}$, the Laplace transform of $I$ is given by the following lemma.

Lemma 1. The Laplace transform of the interference power after canceling the $K$ strongest interferers (conditioned on $y_{K}$ ) is given by

$$
\mathcal{L}_{I}(s)=\mathbb{E}_{I}\left[e^{-s I}\right]=e^{g(s)},
$$

where $g(s)=-2 \pi \lambda\left(g_{L}(s)+g_{N}(s)\right)$ with

$$
\begin{aligned}
& g_{L}(s)=\eta_{L} \int_{y_{K}}^{\infty} \frac{s y^{2 \eta_{L}-1}}{y+s} e^{-\beta y^{\eta_{L}}} \mathrm{~d} y, \\
& g_{N}(s)=\eta_{N} \gamma^{2} \int_{y_{K}}^{\infty} \frac{s y^{2 \eta_{N}-1}}{y+s}\left(1-e^{-\beta \gamma y^{\eta_{N}}}\right) \mathrm{d} y .
\end{aligned}
$$

Proof. Let $g_{k}=\left|\boldsymbol{v}_{0}^{H} \boldsymbol{h}_{k}\right|^{2}$, we have

$$
\begin{gathered}
\mathcal{L}_{I}(s)=\mathbb{E}_{\Phi_{\mathrm{PL}}, g}\left(e^{-s \sum_{k>K} g_{k} y_{k}^{-1}}\right) \\
\stackrel{(a)}{=} \mathbb{E}_{\Phi_{\mathrm{PL}}}\left(\prod_{k>K} \frac{1}{1+s / y_{k}}\right) \\
\stackrel{(b)}{=} e^{-\int_{y_{K}}^{\infty}\left[1-\frac{1}{1+s / y}\right] \lambda(y) \mathrm{d} y},
\end{gathered}
$$

where (a) is due to that $g=\left|\boldsymbol{v}_{0}^{H} \boldsymbol{h}_{k}\right|^{2}$ is iid exponentially distributed with unit mean [16, Lemma 2], and $(b)$ is from the probability generating functional of the PPP [6]. Finally, plugging in the density functions given by (6) and (7) completes the proof.

\section{B. General Coverage Probability}

The coverage probability is defined by the probability that the received SINR is above a threshold $T>0$. In the following, we derive the uplink coverage probability expression. 
Theorem 1. The uplink coverage probability of a $B S$ with $N$ antennas performing PZF and canceling $K, 0 \leq K<N$ strongest interfering signals is given by

$$
\begin{aligned}
& \mathbb{P}(\operatorname{SINR}>T)=\int_{0}^{\infty} \int_{\varphi}^{\infty} e^{g(T \varphi)-T \varphi / S N R_{L}} \sum_{m=0}^{N-K-1} \frac{(-T \varphi)^{m}}{\Gamma(m+1)} \\
& \times B_{m}\left(g^{(1)}(T \varphi)-1 / S N R_{L}, g^{(2)}(T \varphi), \cdots, g^{(m)}(T \varphi)\right) \\
& \times f_{y_{k} \mid y_{0}}\left(y_{K}\right) f_{y_{0}}(\varphi) \mathrm{d} y_{K} \mathrm{~d} \varphi,
\end{aligned}
$$

where the gamma function is defined as $\Gamma(s)=\int_{0}^{\infty} t^{s-1} e^{-t} \mathrm{~d} t$ and $g^{(n)}(s)=-2 \pi \lambda\left(g_{L}^{(n)}(s)+g_{N}^{(n)}(s)\right)$ is the $n$-th derivative with respect to $s$ given by

$$
\begin{aligned}
g_{L}^{(n)}(s)= & (-1)^{n+1} \Gamma(n+1) \eta_{L} \int_{y_{K}}^{\infty} y^{2 \eta_{L}} e^{-\beta y^{\eta_{L}}} \\
& \times(y+s)^{-n-1} \mathrm{~d} y \\
g_{N}^{(n)}(s)= & (-1)^{n+1} \Gamma(n+1) \eta_{N} \gamma^{2} \int_{y_{K}}^{\infty} y^{2 \eta_{N}} \\
& \times\left(1-e^{-\beta \gamma y^{\eta_{N}}}\right)(y+s)^{-n-1} \mathrm{~d} y,
\end{aligned}
$$

with $B_{m}\left(x_{1}, \cdots, x_{m}\right)$ denoting the $m$-th complete exponential Bell polynomial.

Proof. Conditioned on $y_{0}=\varphi$ and $y_{K}$ we have

$$
\begin{aligned}
& \mathbb{P}\left(\operatorname{SINR}>T \mid \varphi, y_{K}\right) \\
= & \mathbb{P}\left(\left|\boldsymbol{v}_{0}^{H} \boldsymbol{h}_{0}\right|^{2}>\left(I+1 / \mathrm{SNR}_{L}\right) T \varphi\right) \\
\stackrel{(c)}{=} & \mathbb{E}_{I}\left(\frac{\Gamma\left(N-K,\left(I+1 / \mathrm{SNR}_{L}\right) T \varphi\right)}{\Gamma(N-K)}\right) \\
= & \mathbb{E}_{I}\left(e^{-\left(I+1 / \mathrm{SNR}_{L}\right) T \varphi} \sum_{m=0}^{N-K-1} \frac{\left[\left(I+1 / \mathrm{SNR}_{L}\right) T \varphi\right]^{m}}{\Gamma(m+1)}\right) \\
= & \left.\sum_{m=0}^{N-K-1} \frac{(-s)^{m}}{\Gamma(m+1)} \frac{\partial^{m}}{\partial s^{m}}\left(\mathcal{L}_{I}(s) e^{-s / \mathrm{SNR}_{L}}\right)\right|_{s=T \varphi}
\end{aligned}
$$

Here, (c) follows from the fact that $\left|\boldsymbol{v}_{0}^{H} \boldsymbol{h}_{0}\right|^{2}$ is distributed as $F(X<x)=\frac{\gamma(N-K, x)}{\Gamma(N-K)}$ [16, Lemma 1], the incomplete gamma functions are defined as $\gamma(s, x)=$ $\int_{0}^{x} t^{s-1} e^{-t} \mathrm{~d} t, \Gamma(s, x)=\int_{x}^{\infty} t^{s-1} e^{-t} \mathrm{~d} t$ and the Laplace transform in the last step is given by Lemma 1. In order to find the $m$-th derivative of the Laplace transform, we use the Faà di Bruno's formula [20] and express the derivatives in terms of Bell polynomials. Thus, the $m$-th derivative of $\mathcal{L}_{(I)}(s) e^{-s / \mathrm{SNR}_{L}}$ is given by

$$
\begin{aligned}
& \frac{\partial^{m}}{\partial s^{m}} \mathcal{L}_{(I)}(s) e^{-s / \mathrm{SNR}_{L}}=\frac{\partial^{m}}{\partial s^{m}} e^{g(s)-s / \mathrm{SNR}_{L}} \\
= & \sum_{k=0}^{m} e^{g(s)-s / \mathrm{SNR}_{L}} \times B_{m, k}\left(g^{(1)}(s)-1 / \mathrm{SNR}_{L}, g^{(2)}(s)\right. \\
& \left.\cdots, g^{(m-k+1)}(s)\right) \\
= & e^{g(s)-s / \mathrm{SNR}_{L}} \times B_{m}\left(g^{(1)}(s)-1 / \mathrm{SNR}_{L}, g^{(2)}(s)\right. \\
& \left.\cdots, g^{(m-k+1)}(s)\right)
\end{aligned}
$$

where the $n$-th derivative of $g_{L}(s)$ and $g_{N}(s)$ with respect to $s$ can be found easily by using the Leibniz integral rule and the last step follows from the definition of the complete exponential Bell polynomial.

Finally, taking expectation over $y_{0}$ and $y_{K}$, we obtain the unconditioned coverage probability expression.

For $N-K \neq 1$, Theorem 1 requires to compute the Bell polynomials and the derivatives of $g(s)$. In the next subsection, we provide a simple upper bound for the coverage probability which does not require the computation of Bell polynomials.

\section{Upper Bound for the Coverage Probability}

Theorem 2. A tight upper bound for the coverage probability is given by

$$
\begin{aligned}
& \mathbb{P}(\operatorname{SINR}>T)<\int_{0}^{\infty} \int_{\varphi}^{\infty} \sum_{n=1}^{N-K}(-1)^{n+1}\left(\begin{array}{c}
N-K \\
n
\end{array}\right) \\
& e^{g(c T \varphi)-c T \varphi / S N R_{L}} f_{y_{k} \mid y_{0}}\left(y_{K}\right) f_{y_{0}}(\varphi) \mathrm{d} y_{K} \mathrm{~d} \varphi,
\end{aligned}
$$

where $c=n[\Gamma(N-K+1)]^{-1 /(N-K)}$.

Proof. Conditioned on $y_{0}=\varphi$ and $y_{K}$, the upper bound is obtained by bounding the incomplete gamma function in (20), and consequently

$$
\begin{aligned}
& \mathbb{P}\left(\operatorname{SINR}>T \mid \varphi, y_{K}\right) \\
\stackrel{(d)}{<} & 1-\mathbb{E}_{I}\left[\left(1-e^{-\Gamma(N-K+1)^{\frac{-1}{N-K}}\left(I+1 / \mathrm{SNR}_{L}\right) T \varphi}\right)^{N-K}\right] \\
\stackrel{(e)}{=} & \sum_{n=1}^{N-K}(-1)^{n+1}\left(\begin{array}{c}
N-K \\
n
\end{array}\right) \mathbb{E}_{I}\left[e^{-c\left(I+1 / \mathrm{SNR}_{L}\right) T \varphi}\right] \\
= & \sum_{n=1}^{N-K}(-1)^{n+1}\left(\begin{array}{c}
N-K \\
n
\end{array}\right) \mathcal{L}_{I}(c T \varphi) e^{-c T \varphi / \mathrm{SNR}_{L}}
\end{aligned}
$$

Here, $(d)$ uses the lower bound for the incomplete gamma function given by $\frac{\gamma(b, x)}{\Gamma(b)}>\left(1-e^{-s_{b} x}\right)^{b}$ [21], where $s_{b}=$ $[\Gamma(1+b)]^{-1 / b}$ for $b>1$ and $(e)$ holds due to the binomial formula. Finally, using Lemma 1 and integrating over $\varphi$ and $y_{K}$ complete the proof. Note that setting $N-K=1$ yields the exact coverage probability for zero-forcing receivers.

\section{Simulation Results}

In this section, we analyze the coverage probability for different network parameters and verify our analytical expressions from Theorem 1 with numerical simulations. We assume a dense network where the average uplink cell range is $r=10$ $\mathrm{m}$, so that the density of users sharing the same frequency is given by $\lambda=1 /\left(\pi r^{2}\right)$. The additive noise power is given by $\sigma^{2}=-174 \mathrm{dBm}+10 \log _{10}(\mathrm{~B})+\mathrm{NF}$, where B is the bandwidth and $\mathrm{NF}$ is the noise figure in $\mathrm{dB}$. We assume $\mathrm{B}=1 \mathrm{GHz}$, $\mathrm{NF}=10 \mathrm{~dB}$ and the transmit power $P=30 \mathrm{dBm}$, unless otherwise stated. The path loss parameters used in this section are $\alpha_{L}=2.1, \alpha_{N}=3.17, C_{L}=-60 \mathrm{~dB}$ and $C_{N}=-70 \mathrm{~dB}$, which are typical for micro-cellular systems operating at 2 to 


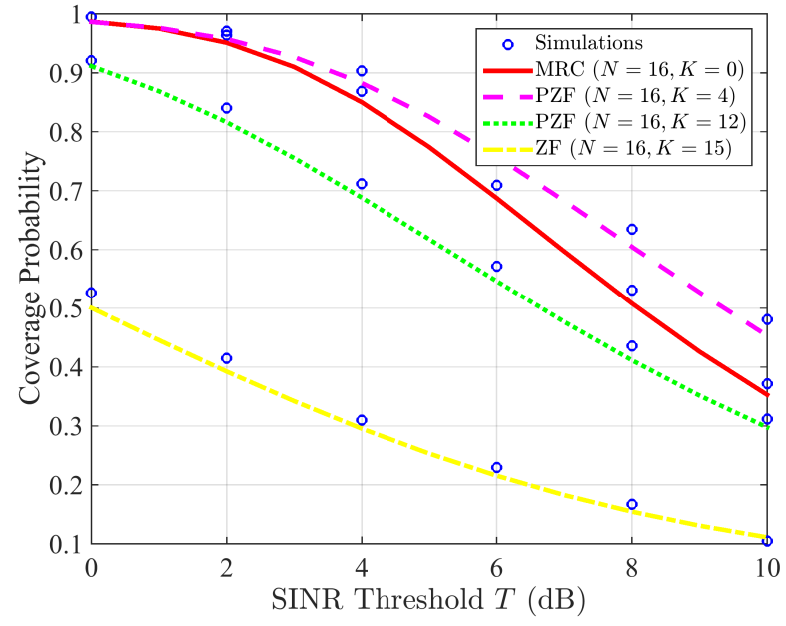

Figure 2. Coverage probability based on Theorem 1 and simulations. The results are shown for different antenna configurations.

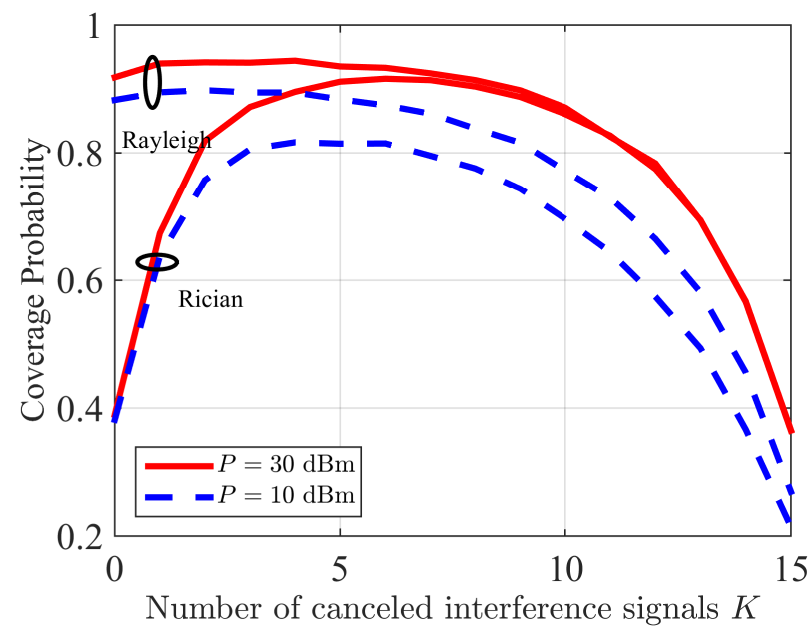

Figure 3. Uplink coverage probability for the PZF receiver versus the number of cancelled interferers $K$ for Rayleigh fading and Rician fading in LOS signals, the NLOS signals are assumed to have Rayleigh fading. Here, the SINR threshold is $T=3 \mathrm{~dB}, N=16$ and $K_{\text {Rician }}=1$.

$73 \mathrm{GHz}$ [22] and the LOS probability parameter is given by $\beta=0.01$.

In Fig. 2, Monte Carlo simulations are shown by generating interference channels from randomly dropped users and the results are compared with the analytical results from Theorem 1. As seen, Theorem 1 can predict the coverage probability in dense mmWave networks with high accuracy but with much less computation complexity than system simulations. By comparing the coverage probability for different antenna configurations, we notice that the coverage probability at a given SINR threshold first increases with $K$ and then decreases for large $K$, while the $\mathrm{ZF}$ receiver gives the worst performance among all configurations. This point can be seen more clearly in Fig. 3 where we observe a large coverage difference between the ZF receiver and the PZF receiver with

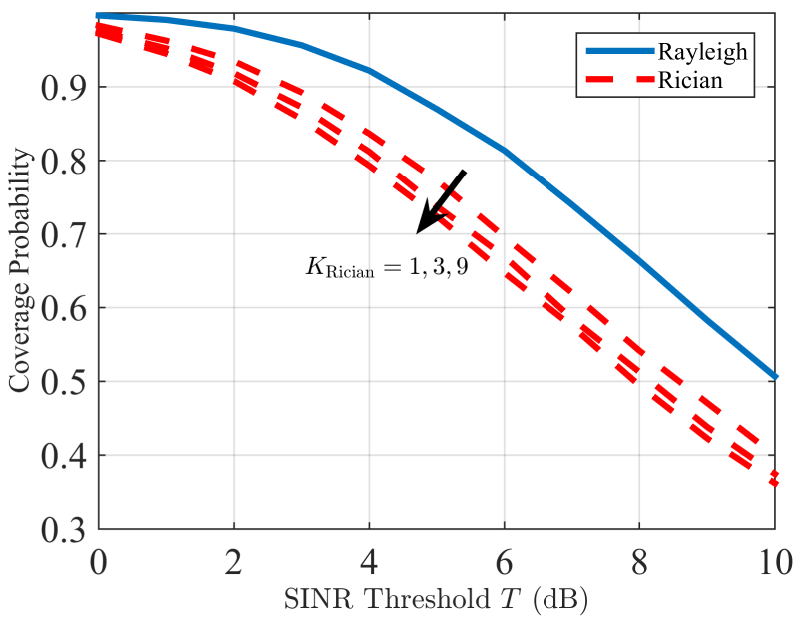

Figure 4. Coverage probability for Rician fading in LOS links, $N=16$ and $K=4$.

an optimal $K$ maximizing the coverage probability. The results indicate that using multiple antennas' degrees of freedom for interference cancellation is less effective than for boosting the useful signal and exploiting the array gain. This is because the high attenuation property of mmWave signals helps with interference cancellation and it is more likely that there are only a small number of strong interferers.

In Fig. 3, we show the effects of reducing the transmit powers of both the useful signal and the interference signals. The results show that the coverage probability decreases when the transmit power is reduced. Intuitively, this is due to the fact that the useful signal power decreases faster than the interference power, as the reduced amount in NLOS interfering signals is negligible.

To compare our analytical work with the case of Rician fading, in Fig. 3-4, we numerically evaluate the coverage probability with Rician fading in LOS links and Rayleigh fading in NLOS links and label these curves with Rician. The Rician fading coefficients are generated according to $h_{\text {Rician }}=X+i Y$, where $X \sim \mathcal{N}\left(v \cos \theta, \sigma^{2}\right), Y \sim$ $\mathcal{N}\left(v \sin \theta, \sigma^{2}\right), K_{\text {Rician }}=\frac{v^{2}}{2 \sigma^{2}}$ is the ratio between the power in the direct path and the power in the scattered paths and $\theta$ is a real number. Note that curves labeled with Rayleigh correspond to the case of Rayleigh fading in both LOS and NLOS links. From Fig. 4, we see that Rician fading results in increased interference power and leads to lower coverage probability, compared to Rayleigh fading. Hence, we need to use more antennas for interference cancellation, compared to the case with Rayleigh fading. This is confirmed in Fig. 3 where we plot the case with Rician fading for $K_{\text {rician }}=1$ and different antenna configurations. In the case of Rician fading, we find that the optimal $K$ is larger than that of Rayleigh fading and there is noticeable performance difference between the MRC receiver and the ZF receiver even for small $T$, which further confirms the strength of PZF receivers in the presence of strong interference signals. Also, this observation 


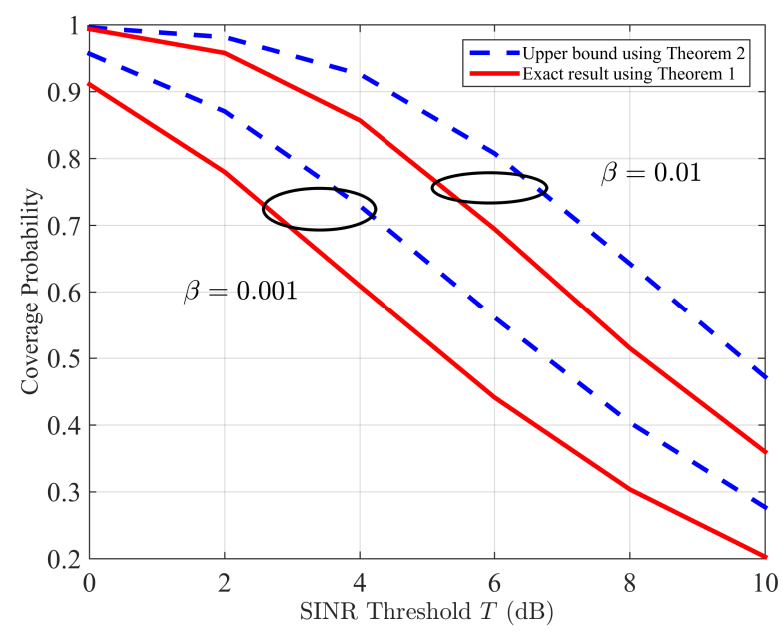

Figure 5. Upper bound of the coverage probability for the PZF receiver with $N=16, K=0$.

shows how to best use antennas in mmWave networks, the trade-off between interference cancellation and useful signal enhancement should be determined according to the channel's fading and blockage environment.

In Fig. 5, we plot the exact coverage probability, found by Theorem 1 and verified by simulations, as well as its upper bound for different values of $\beta$ which determines how fast the LOS probability decays as the distance increases (see (1) and its corresponding explanations). As shown for all the considered thresholds, the gap between the upper bound and Theorem 1 is less than $2 \mathrm{~dB}$. Also, the figure shows that the coverage probability increases as the LOS probability decreases, because of the increased number of NLOS interferers.

\section{CONCLUSiON}

This paper analyzes the uplink coverage probability in a mmWave cellular system where users are equipped with single antennas and BSs utilize multi-antenna PZF receivers for useful signal enhancement and interference cancellation. Considering a LOS probability function-based path loss model, the coverage probability is shown to be maximized when using a subset of antennas' degree-of-freedom for useful signal enhancement and using the remaining degrees of freedom for canceling the interference from strongest interferers. Due to the blockage effects and the high attenuation of mmWave signals, the number of strong interferers is small, thus most of antennas' degrees of freedom should be used for useful signal enhancement. Particularly, compared to zero-forcing, the PZF scheme is shown to improve the coverage probability significantly.

\section{ACKNOWLEDGMENT}

The research leading to these results received funding from the European Commission H2020 programme under grant agreement $n^{\circ} 671650$ (5G PPP mmMAGIC project), and from the Swedish Governmental Agency for Innovation Systems (VINNOVA) within the VINN Excellence Center Chase.

\section{REFERENCES}

[1] T. Rappaport, S. Sun, R. Mayzus, H. Zhao, Y. Azar, K. Wang, G. Wong, J. Schulz, M. Samimi, and F. Gutierrez, "Millimeter wave mobile communications for 5G cellular: It will work!" IEEE Access, vol. 1, pp. 335-349, 2013.

[2] F. Boccardi, R. Heath, A. Lozano, T. Marzetta, and P. Popovski, "Five disruptive technology directions for 5G," IEEE Commun. Mag., vol. 52, no. 2, pp. 74-80, February 2014.

[3] J. Andrews, S. Buzzi, W. Choi, S. Hanly, A. Lozano, A. Soong, and J. Zhang, "What will 5G be?" IEEE J. Sel. Areas Commun, vol. 32, no. 6, pp. 1065-1082, June 2014.

[4] Ericsson, "Ericsson mobility report," Available: http://www.ericsson.com/res/docs/2015/ericsson-mobility-report-june2015.pdf, June 2015.

[5] M. K. Samimi, G. R. MacCartney, S. Sun, and T. S. Rappaport, " $28 \mathrm{GHz}$ millimeter-wave ultrawideband small-scale fading models in wireless channels," in 2016 IEEE 83rd Vehicular Technology Conference (VTC Spring), May 2016, pp. 1-6.

[6] M. Haenggi, Stochastic geometry for wireless networks. Cambridge : Cambridge University Press, 2013.

[7] J. Andrews, F. Baccelli, and R. Ganti, "A tractable approach to coverage and rate in cellular networks," IEEE Trans. Commun., vol. 59, no. 11, pp. 3122-3134, November 2011.

[8] T. Bai, R. Vaze, and R. W. Heath, "Analysis of blockage effects on urban cellular networks," IEEE Trans. Wireless Commun., vol. 13, no. 9, pp. 5070-5083, Sept 2014.

[9] M. N. Kulkarni, S. Singh, and J. G. Andrews, "Coverage and rate trends in dense urban mmwave cellular networks," in GLOBECOM, Austin, TX, USA, Dec 2014, pp. 3809-3814.

[10] T. Bai and R. W. Heath, "Coverage and rate analysis for millimeter-wave cellular networks," IEEE Trans. Wireless Commun., vol. 14, no. 2, pp. 1100-1114, Feb 2015.

[11] C. Galiotto, N. Pratas, N. Marchetti, and L. Doyle, "A stochastic geometry framework for LOS/NLOS propagation in dense small cell networks," in ICC, London, UK, June 2015, pp. 2851-2856.

[12] T. Novlan, H. Dhillon, and J. Andrews, "Analytical modeling of uplink cellular networks," IEEE Trans. Wireless Commun., vol. 12, no. 6, pp. 2669-2679, June 2013.

[13] S. Singh, X. Zhang, and J. G. Andrews, "Joint rate and SINR coverage analysis for decoupled uplink-downlink biased cell associations in HetNets," IEEE Trans. Wireless Commun., vol. 14, no. 10, pp. 53605373, Oct 2015.

[14] T. Novlan and J. Andrews, "Analytical evaluation of uplink fractional frequency reuse," IEEE Trans. Commun., vol. 61, no. 5, pp. 2098-2108, May 2013.

[15] T. Bai, V. Desai, and R. Heath, "Millimeter wave cellular channel models for system evaluation," in ICNC, Honolulu, Hawaii, USA, Feb 2014, pp. $178-182$.

[16] N. Jindal, J. Andrews, and S. Weber, "Rethinking MIMO for wireless networks: Linear throughput increases with multiple receive antennas," in ICC, Dresden, Germany, June 2009, pp. 1-6.

[17] S. Veetil, K. Kuchi, and R. Ganti, "Performance of PZF and MMSE receivers in cellular networks with multi-user spatial multiplexing," IEEE Trans. Wireless Commun., vol. 14, no. 9, pp. 4867-4878, Sept 2015.

[18] T. Bai and R. Heath, "Coverage analysis for millimeter wave cellular networks with blockage effects," in GlobalSIP, Austin, TX, Dec 2013, pp. 727-730.

[19] D. Moltchanov, "Distance distributions in random networks," Ad Hoc Networks, vol. 10, no. 6, pp. 1146-1166, 2012.

[20] W. P. Johnson, "The curious history of Faa di Bruno's formula," The American Mathematical Monthly, vol. 109, pp. 217-234, 2002.

[21] W. Gautschi, "The incomplete gamma function since Tricomi," Atti dei Convegni Lincei, Accademia Nazionale dei Lincei, Roma,, vol. 147, pp. 203-237, 1998.

[22] Millimetre-Wave Based Mobile Radio Access Network for Fifth Generation Integrated Communications (mmMAGIC), "Measurement campaigns and initial channel models for preferred suitable frequency ranges," March 2016. [Online]. Available: https://bscw.5g-mmmagic. eu/pub/bscw.cgi/d94832/mmMAGIC\$\\$D2-1.pdf 